\title{
Une analyse spatiale des mouvements de l'emploi et de la population en France
}

A Spatial Analysis of Jobs and Population Flows in France

\section{Émilie Arnoult et Richard Duhautois}

\section{OpenEdition}

\section{Journals}

Édition électronique

URL : http://journals.openedition.org/travailemploi/9502

DOI : $10.4000 /$ travailemploi.9502

ISSN : 1775-416X

Éditeur

DARES - Ministère du Travail

\section{Édition imprimée}

Date de publication : 2 décembre 2019

Pagination : 23-46

ISSN : 0224-4365

\section{Référence électronique}

Émilie Arnoult et Richard Duhautois, « Une analyse spatiale des mouvements de l'emploi et de la population en France », Travail et Emploi [En ligne], 160 | 2019, mis en ligne le 01 novembre 2020, consulté le 25 mars 2021. URL : http://journals.openedition.org/travailemploi/9502 ; DOI : https:// doi.org/10.4000/travailemploi.9502 


\title{
Une analyse spatiale des mouvements de l'emploi et de la population en France
}

\author{
Émilie Arnoult ${ }^{*}$ Richard Duhautois ${ }^{* *}$
}

La mobilité résidentielle des ménages et la localisation des activités économiques sont des sujets souvent abordés dans la littérature, qui s'interroge sur leurs effets sur les disparités entre les territoires. Les dynamiques de l'emploi et de la population paraissent étroitement liées, et peuvent par exemple conduire à une concentration de la population et des activités dans certains territoires, quand d'autres voient leur niveau d'emploi et de population reculer. Afin d'approfondir les connaissances sur le sujet, nous proposons dans cet article d'explorer la complexité du lien entre la mobilité résidentielle et la croissance locale de l'emploi salarié privé. Pour cela, nous supposons que les dynamiques locales de l'emploi et de la population peuvent varier selon les profils socioéconomiques des ménages et selon la nature des contrats de travail signés. À partir des données du Recensement de la population et des DMMO-EMMO (Déclarations mensuelles des mouvements de main-d'œuvre - Enquêtes sur les mouvements de main-d'œuvre), nous montrons que la dynamique des emplois en contrat à durée déterminée (CDD) est très fortement liée aux mouvements de la population, en particulier ceux des personnes peu qualifiées. À l'inverse, la dynamique résidentielle, quelles que soient les professions et catégories socioprofessionnelles, est uniquement influencée par les mouvements d'emploi en contrat à durée indéterminée (CDI).

$\mathrm{L}$ a localisation résidentielle des ménages et celle des activités économiques sont des sujets souvent abordés dans la littérature. Leurs dynamiques semblent étroitement liées, et la compréhension du sens de causalité entre les évolutions de la population et celles de l'emploi représente un enjeu pour les décideurs publics. Si l'emploi suit la population, les territoires souhaitant se dynamiser ont tout intérêt à investir dans les équipements qui les rendent attractifs aux yeux de la population ; les personnes les plus qualifiées notamment sont sensibles à la présence d'aménités sur les territoires, et leur

\footnotetext{
* Cnam-Lirsa et CEET ; emilie.arnoult@lecnam.net.

**Cnam-Lirsa et CEET ; richard.duhautois@lecnam.net.
} 
installation peut conduire à la hausse de l'emploi alentour. À l'inverse, si la population suit l'emploi, les personnes qui déménagent cherchent à se rapprocher des lieux où les perspectives d'emploi sont favorables. Le fait que la population recherche un emploi stable peut conduire les décideurs publics à créer un cadre propice au développement des activités économiques pour attirer les entreprises, et indirectement la population.

Historiquement, la mobilité résidentielle est étroitement liée à la conjoncture économique (BACCAÏNI et al., 2018). La crise des années 1970 entraîna ainsi une augmentation de la mobilité résidentielle en France : les personnes privées involontairement d'emploi déménageaient pour se rapprocher des bassins d'emploi plus dynamiques et augmenter leurs chances de retour à l'emploi. Si les mobilités résidentielles des années 1970 laissent penser que, à la suite du choc économique, la population a suivi l'emploi, les comportements observés après la crise de 2008 ne semblent pas suivre la même logique. Entre 1990 et 2014, on observe un ralentissement de la mobilité résidentielle, et en particulier celle de longue distance : la part des déménagements ayant eu lieu entre départements est passée de $34 \%$ à $31 \%$ (BACCAÏNI et al., 2018).

L'objectif de ce travail est justement d'étudier les comportements migratoires en lien avec les dynamiques de l'emploi qui ont suivi la crise de 2008. Il existe un grand nombre de travaux basés sur le modèle de Gerald A. CARLINo et Edwin S. MiLLs (1987), qui étudient le lien entre les dynamiques locales de l'emploi et de la population. Cependant, les résultats des études empiriques varient beaucoup selon les données mobilisées et selon l'échelle géographique à laquelle les études sont menées (HoOGSTRA et al., 2017). Nous souhaitons dans ce travail déterminer si c'est la population qui suit l'emploi ou bien si c'est l'emploi qui suit la population, en faisant l'hypothèse qu'il existe une hétérogénéité au sein des dynamiques de l'emploi et de celles de la population. Nous supposons que même si les comportements migratoires (de mobilité résidentielle) sont étroitement liés aux dynamiques du marché du travail local, ils ne sont pas homogènes au sein de la population. La catégorie socioprofessionnelle des ménages peut jouer un rôle important sur leur propension à être mobiles : les personnes qualifiées déménagent plus souvent que les personnes non qualifiées, et ont plus souvent tendance à changer de région de résidence (GOBILLON, 2001 ; DeBrand, TAFFin, 2005). Par ailleurs, nous supposons que la propension à la mobilité résidentielle ne dépend pas de l'évolution de l'emploi dans son ensemble, mais qu'elle est plus fortement liée aux contrats de longue durée. En ce sens, les choix de localisation résidentielle seraient davantage influencés par les embauches en contrats à durée indéterminée (CDI) que par la dynamique des contrats courts.

Contrairement aux modèles estimés à partir des taux de variation de l'emploi de la population, nous distinguons dans ce travail les mouvements d'entrées de populations sur un territoire donné et les mouvements de sorties de ce territoire. Cette distinction permet d'analyser plus finement le lien entre les dynamiques locales de l'emploi (dans le secteur privé) et de la population, et ainsi de mieux appréhender la complexité de cette relation. Cette méthode a notamment montré que bien que la croissance de l'emploi et celle de la population s'influencent mutuellement, les mouvements d'entrées impulsent fortement les dynamiques jointes de l'emploi et de la population, alors que les sorties 
de populations ne sont pas sensibles aux sorties d'emploi (ARnoult, Duhautois, 2019). Nous prolongeons ici l'analyse en supposant qu'il peut aussi y avoir des écarts de comportement selon les caractéristiques des personnes et des emplois.

Nous analysons les déménagements des populations en âge de travailler entre 2012 et 2013 à partir de deux sources : le Recensement de la population de 2013 (Institut national de la statistique et des études économiques, Insee), et celle des mouvements de main-d'œuvre sur la même période (Déclarations mensuelles des mouvements de main-d'œuvre [DMMO] - Enquête sur les mouvements de main-d'œuvre [EMMO] de 2012, pilotées par la Direction de l'animation de la recherche, des études et des statistiques, Dares). Si la fenêtre temporelle d'une année peut paraître courte pour appréhender des dynamiques susceptibles d'avoir des effets sur un plus long terme, les données que nous mobilisons ont l'avantage de fournir des informations précises sur les caractéristiques des individus et des mouvements d'emploi. Elles nous permettent notamment de nous focaliser sur les individus qui décident de leur mobilité (qu'ils se déclarent chefs de ménages ou conjoints, âgés de 15 à 64 ans ${ }^{1}$ ). Nous distinguons les mouvements d'emploi selon qu'ils se traduisent par des CDD (contrat à durée déterminée) ou des CDI (contrat à durée indéterminée). Cette distinction nous semble d'autant plus nécessaire que les embauches se font en CDD dans $87 \%$ des cas, et qu'il peut s'agir de contrats très courts puisque $30 \%$ des CDD signés le sont pour une durée d'un jour (MiLIN, 2018). Nos résultats semblent en effet montrer que le lien de causalité entre les dynamiques de l'emploi et celles de la population est plus complexe que ce que tend à établir la littérature. Il varie fortement selon les caractéristiques du contrat de travail et les professions et catégories socioprofessionnelles (PCS), mais aussi selon que l'on distingue, ou non, les mouvements d'entrées des mouvements de sorties.

Dans la suite de cet article, nous exposons les mécanismes de la relation entre localisation de la population et emploi mis en évidence dans la littérature. Nous nous consacrons ensuite à la présentation de la méthode empirique, puis à celle, détaillée, des données et des résultats.

\section{Revue de la littérature}

La littérature a mis en évidence depuis longtemps la relation étroite existant entre la localisation de la population et celle de l'emploi et a montré en quoi leurs caractéristiques respectives expliquent les écarts observés entre les territoires. Cette partie décrit dans un premier temps les mécanismes de cette relation puis, dans un second temps, la façon dont nos résultats complètent la compréhension que l'on peut en avoir.

1. Pour le Bureau international du travail (BIT), la population en âge de travailler est celle âgée de plus de 15 ans, sans limite supérieure. Par convention, les statistiques portent toutefois souvent sur la tranche 15-64 ans et c'est celle retenue ici. 


\section{Localisation de la population et localisation de l'emploi}

De façon générale, la mobilité résidentielle est présentée comme un processus de choix en deux étapes : le choix de mobilité puis le choix de localisation. Cette seconde étape dépend des raisons qui ont motivé la mobilité (DEBRAND, TAFFIn, 2005). Si elle est due à une modification de la composition du ménage, la mobilité résidentielle est le plus souvent de courte distance : elle a lieu au sein de la commune ou dans une commune voisine, et se produit de façon relativement indépendante des questions liées à l'emploi (AUTHIER et al., 2010). À l'inverse, les mobilités résidentielles en lien avec un changement au niveau de l'emploi sont plus souvent de longue distance et peuvent dès lors entraîner un changement du cadre de vie.

Le choix de localisation résidentielle dépend de deux éléments : l'emploi et les caractéristiques du territoire. La distance au marché du travail local détermine le lieu de résidence des ménages dans le modèle standard de l'économie urbaine, mais l'offre différenciée d'aménités non marchandes peut aussi expliquer que les ménages s'installent dans une localité plutôt que dans une autre. Le choix de localisation ne dépend donc pas seulement de la distance à l'emploi, elle dépend aussi des caractéristiques de chaque zone, qu'il s'agisse de la présence d'aménités naturelles et environnementales, de l'offre de transports, des caractéristiques du marché du logement, de la qualité des biens publics locaux, de l'offre culturelle locale, de l'environnement social, etc. (BrueCKNer et al., 1999 ; HuU Phe, Wakely, 2000 ; Kim et al., 2005). Finalement, seul un tiers des déménagements en France ont lieu entre des départements distincts (BACCAÏNI et al., 2018), et ce sont ces mobilités de longue distance qui sont le plus en lien avec les dynamiques de l'emploi, l'emploi jouant un rôle moindre dans les mobilités de plus courte distance.

Les travaux de la nouvelle économie géographique montrent que les entreprises choisissent leur localisation de façon à minimiser leurs coûts de production et à accroître leurs rendements. Selon Paul Krugman et ses coauteurs (HelPMAn, Krugman, 1985 et KRUGMan, VANABLES, 1990), la concentration des activités productives permet de réduire les coûts liés aux transports de biens intermédiaires ; elle favorise également une meilleure diffusion des connaissances, augmentant ainsi la productivité des salariés. L'agglomération des activités dépend cependant du degré de mobilité des travailleurs (PuGA, 1999). Si les individus sont mobiles, les entreprises se regroupent en proposant des salaires plus élevés qu'ailleurs afin de pouvoir bénéficier des effets positifs de l'agglomération. À l'inverse, si la propension à la mobilité des travailleurs est faible, les entreprises tendent à se disperser sur le territoire. Dans les faits, il existe évidemment des individus mobiles et d'autres qui le sont moins, ce qui aboutit à un double modèle de localisation des entreprises : à la fois de concentration et de dispersion. Ce modèle dépend notamment des catégories sociales : les cadres, plus mobiles, se retrouvent essentiellement dans les agglomérations et les employés et ouvriers dans des zones périurbaines ou rurales (PUGA, 1999). 


\section{Enseignements de la littérature sur les dynamiques jointes de l'emploi et de la population}

Les travaux cités ci-dessus ne permettent pas d'étudier de façon jointe la localisation des entreprises et celle de la population. G. A. CARLino et E. S. Mills (1987) ont quant à eux développé un modèle qui permet d'analyser, à l'échelle locale et en tenant compte des spécificités des territoires, les dynamiques jointes de l'emploi et de la population. Ce modèle a donné lieu à un grand nombre d'applications, mais celles-ci ne permettent pas d'aboutir à un consensus sur le lien de causalité entre les évolutions de la population et celles de l'emploi (HoOgSTRA et al. 2017). D'une part, la population peut suivre l'emploi : le choix de la localisation résidentielle est déterminé par sa proximité avec un marché de l'emploi dynamique. D'autre part, l'emploi peut suivre la population ; les entreprises choisissent de s'implanter à proximité de la population, qui représente pour elles aussi bien des consommateurs - donc une source de débouchés pour leurs produits - qu'une main-d'œuvre nécessaire à leur production.

Ces travaux ont été menés sur différents pays, et les 321 résultats provenant de 64 études varient fortement selon les choix de modélisation réalisés par les auteurs, mais ils concluent que, globalement, ce sont plutôt les emplois qui suivent la population que l'inverse (HoOGSTRA et al., 2017). Quelques applications récentes prennent en compte l'hétérogénéité de la population et de l'emploi, et son incidence sur les évolutions observées. Ainsi, la relation entre les dynamiques de l'emploi et de la population dépend des secteurs d'emploi considérés, selon qu'il s'agit d'emplois qui relèvent de la sphère résidentielle ou de la sphère productive ${ }^{2}$. Des travaux menés sur données danoises (ABILDTRUP et al., 2012), néerlandaises (DE GRAAFF et al., 2012) ou françaises (ABILDTRUP et al., 2018) confirment que l'emploi relevant de la sphère résidentielle suit la population, mais que la population suit l'emploi qui relève de la sphère productive. Stein ØSTBYE et ses co-auteurs (2018) poussent plus loin l' analyse multisectorielle, en distinguant les emplois et les individus selon leur niveau de qualification. En estimant un modèle à équations simultanées, ils montrent que l'emploi qualifié suit la population qualifiée, et qu'il existe aussi un effet d'accumulation des emplois : la croissance de l'emploi qualifié entraîne celle de l'emploi non qualifié, et réciproquement.

Notre étude s'inscrit dans le prolongement de ce travail. Nous souhaitons vérifier si les interactions entre les mouvements d'emploi et de populations sont identiques selon qu'il s'agit d'emplois contractés pour une durée déterminée ou indéterminée. Par ailleurs, nous distinguons les individus selon leurs professions et catégories socioprofessionnelles afin de tester l'hypothèse selon laquelle les profils socioéconomiques des ménages ont un impact sur les comportements migratoires. Il a en effet été montré que les trajectoires résidentielles varient fortement selon ces profils (BRIARD, 2019).

2. Selon l'Insee, la sphère productive regroupe les activités orientées vers les marchés extérieurs (nationaux ou internationaux), alors que la sphère de l'économie résidentielle concerne principalement les services aux particuliers, la construction, la santé, l'action sociale, le commerce de détail, les activités financières et le transport de voyageurs (JEAN, 2008). 


\section{Stratégie empirique}

G. A. CARLINo et E. S. MiLlS (1987) proposent un modèle à deux équations simultanées qui permet d'étudier l'interdépendance entre l'évolution de la population et l'évolution de l'emploi. Ce modèle traduit l'hypothèse selon laquelle la localisation des personnes et celle des entreprises s'influencent mutuellement. La mobilité des individus dépend de leur revenu et de leur consommation d'un ensemble de biens et de services marchands ou non marchands. Les individus choisissent la localisation qui maximise leur bien-être, c'est-à-dire celle qui leur offre l'accès au plus grand nombre de biens et services, tout en tenant compte de leurs revenus et de la distance à leur lieu de travail. Quant aux entreprises, elles s'établissent dans des localités qui leur permettent de réduire leurs coûts de production et de se rapprocher de marchés locaux dynamiques, aussi bien du point de vue de la main-d'œuvre qu'en ce qui concerne les débouchés. Dès lors, il convient de tenir compte des spécificités des territoires susceptibles d'influencer la localisation des ménages et des entreprises. En étudiant l'évolution des disparités entre les communes du New Jersey, Marlon G. BOARNET (1994) montre qu'il peut exister des effets de débordement entre des zones géographiques voisines. Un individu peut travailler dans une zone et résider dans une autre, tout comme les débouchés d'une entreprise peuvent ne pas se limiter à la localité où elle est implantée. L'existence de cette dépendance spatiale nécessite l'utilisation d'outils adaptés à l'étude de données agrégées au niveau des localités. Nous estimons le modèle de G. A. CARLINO et E. S. Mills (1987) en mobilisant non pas les variations ou les taux de croissance de la population et de l'emploi, mais en mobilisant directement les flux de population et d'emploi, c'est-à-dire les mouvements d'entrées et de sorties des zones d'emploi.

\section{Présentation du modèle économétrique}

Le modèle classique de G. A. CARLino et E. S. Mills (1987) suppose que, à terme, pour chaque zone, il existe un niveau d'emploi et un niveau de population d'équilibre tels que :

$$
\left\{\begin{array}{l}
E_{i, t}^{*}=f\left(P_{i, t}, X_{i, t-1}^{e m p}\right) \\
P_{i, t}^{*}=f\left(E_{i, t}, X_{i, t-1}^{\text {pop }}\right)
\end{array}\right.
$$

L'emploi d'équilibre d'une zone (noté $E^{*}$ ) dépend du niveau de population observé (noté $P$ ) et des caractéristiques propres au territoire qui jouent un rôle sur l'emploi $\left(X^{e m p}\right)$ telles que la composition de la main-d'œuvre, la répartition sectorielle des emplois, la présence de services aux entreprises, etc. La population d'équilibre d'une zone $\left(P^{*}\right)$ dépend du niveau d'emploi observé ainsi que des facteurs qui influencent la localisation de la population notés $X^{p o p}$ (l'offre de logements, la présence d'aménités, etc.). Dans le modèle d'origine, les facteurs territoriaux sont considérés comme exogènes et ne sont pas influencés par le temps qui passe : on suppose que les caractéristiques territoriales 
sont relativement stables à court terme. Par précaution, ces variables sont usuellement introduites à la période $t-1$ dans le modèle afin de limiter le biais de simultanéité.

La variation des niveaux d'emploi et de population observée entre deux dates va dépendre de l'éloignement de leurs valeurs d'équilibres, les paramètres $\lambda_{E}$ et $\lambda_{P}$ décrivant leur vitesse d'ajustement. Par substitution, on obtient le système d'équations suivant :

$$
\left\{\begin{array}{l}
\Delta \mathrm{E}_{i, t}=E_{i, t}-E_{i, t-1}=\lambda_{E}\left(E_{i}^{*}-E_{i, t-1}\right) \\
\Delta \mathrm{P}_{i, t}=P_{i, t}-P_{i, t-1}=\lambda_{P}\left(P_{i}^{*}-P_{i, t-1}\right)
\end{array}\right.
$$

où $\Delta \mathrm{E}_{i, t}$ et $\Delta \mathrm{P}_{i, t}$ correspondent respectivement à la variation de l'emploi et à la variation de la population mesurées entre deux périodes $t-1$ et $t$.

Par des effets de substitution entre les équations (1) et (2), et en supposant que les relations exprimées dans le système d'équations (1) sont de forme linéaire, l'estimation empirique du modèle classique de G. A. Carlino et E. S. Mills (1987) s'écrit usuellement comme suit :

$$
\left\{\begin{array}{c}
\Delta \mathrm{E}_{i, t}=\alpha_{0}+\alpha_{1} \Delta \mathrm{P}_{i, t}+\alpha_{2} P_{i, t-1}-\lambda_{E} E_{i, t-1}+\sum_{e m p=1}^{K_{1}} \alpha_{e m p} X_{i, t-1}^{e m p}+v_{i t} \\
\Delta \mathrm{P}_{i, t}=\beta_{0}+\beta_{1} \Delta \mathrm{E}_{i, t}+\beta_{2} E_{i, t-1}-\lambda_{P} P_{i, t-1}+\sum_{p o p=1}^{K_{2}} \beta_{p o p} X_{i, t-1}^{p o p}+\mu_{i t}
\end{array}\right.
$$

M. G. BoARNET (1994) a montré la nécessité de tenir compte de la dépendance spatiale entre les différentes localités : les évolutions de l'emploi et de la population peuvent ne pas résulter uniquement des dynamiques internes à chaque localité, mais suivre une évolution plus régionale. En d'autres termes, les dynamiques d'une zone donnée peuvent être liées aux évolutions observées dans les zones géographiques voisines. On note $W$ la matrice qui décrit la relation de voisinage entre chaque zone $i$ et l'ensemble des autres unités. Elle est de taille $N \times N$ et de diagonale nulle, une zone ne pouvant être considérée comme sa propre voisine $\left(W_{i i}=0\right)$. Les équilibres de l'emploi et de la population de long terme peuvent s'écrire :

$$
\left\{\begin{array}{l}
E_{i, t}^{*}=f\left((I+W) P_{i, t}, W E_{i, t}, X_{i, t-1}^{e m p}\right) \\
P_{i, t}^{*}=f\left((I+W) E_{i, t}, W P_{i, t}, X_{i, t-1}^{\text {pop }}\right)
\end{array}\right.
$$

avec $W E_{i, t}$ et $W P_{i, t}$ désignant les niveaux d'emploi et de population observés dans la région. En supposant que les vitesses d'ajustement à leur valeur d'équilibre sont identiques entre les zones et par substitution, le modèle s'écrit :

$$
\left\{\begin{array}{c}
\Delta P_{i, t}=\alpha_{0}+\alpha_{1} \Delta \mathrm{E}_{i, t}+\alpha_{2} W \Delta E_{i, t}+\alpha_{3} W \Delta P_{i, t}+\alpha_{4} P_{i, t-1}+\alpha_{5} E_{i, t-1}+\sum_{e m p=1}^{K_{1}} \alpha_{e m p} X_{i, t-1}^{e m p}+\mu_{i t} \\
\Delta E_{i, t}=\beta_{0}+\beta_{1} \Delta \mathrm{P}_{i, t}+\beta_{2} W \Delta P_{i, t}+\beta_{3} W \Delta E_{i, t}+\beta_{4} E_{i, t-1}+\beta_{5} P_{i, t-1}+\sum_{p o p=1}^{K_{2}} \beta_{p o p} X_{i, t-1}^{p o p}+v_{i t}
\end{array}\right.
$$

Par ailleurs, il peut également exister une forme de dépendance spatiale entre les termes d'erreur des deux équations (GEBREMARiam et al., 2008). Afin d'en tenir 
compte, ces termes d'erreur se décomposent en une partie aléatoire et en une partie afférente à la dépendance spatiale entre les observations :

$$
\left\{\begin{array}{l}
\mu_{i t}=\rho_{1} W \cdot \mu_{i t}+\varepsilon_{i t} \\
v_{i t}=\rho_{2} W \cdot v_{i t}+\epsilon_{i t}
\end{array}\right.
$$

Pour adapter le modèle décrit dans le système d'équations (5) à l'utilisation de données de flux, on suppose que les variations de l'emploi et de la population au cours d'une année résultent de la différence entre les flux d'entrées et les flux de sorties au cours de l'année, de telle sorte que $\Delta \mathrm{P}_{i}=\mathrm{EP}_{i, t}-\mathrm{SP}_{i, t}$ et $\Delta \mathrm{E}_{i}=\mathrm{EE}_{i, t}-\mathrm{SE}_{i, t}$ avec EP les entrées de population, SP les sorties de population, EE les entrées d'emploi et SE les sorties d'emploi. Par substitution, on obtient deux modèles d'équations simultanées, le premier concernant les flux d'entrées et le second les flux de sorties :

$$
\begin{aligned}
& \left\{\begin{aligned}
\mathrm{EP}_{i, t} & =\alpha_{0}^{\prime}+\alpha_{1}^{\prime} \mathrm{EE}_{i, t}+\alpha_{2}^{\prime} W \mathrm{EE}_{i, t}+\alpha_{3}^{\prime} W \mathrm{EP}_{i, t}+\alpha_{4}^{\prime} \mathrm{SP}_{i, t}+\alpha_{5}^{\prime} W \mathrm{SP}_{i, t}+\alpha_{6}^{\prime} \mathrm{SE}_{i, t} \\
& +\alpha_{7}^{\prime} W \mathrm{SE}_{i, t}+\alpha_{8}^{\prime} P_{i, t-1}+\alpha_{9}^{\prime} E_{i, t-1}+\sum_{e m p=1}^{K_{1}} \alpha_{e m p} X_{i, t-1}^{e m p}+\mu_{i, t} \\
\mathrm{EE}_{i, t} & =\beta_{0}^{\prime}+\beta_{1}^{\prime} \mathrm{EP}_{i, t}+\beta_{2}^{\prime} W \mathrm{EP}_{i, t}+\beta_{3}^{\prime} W \mathrm{EE}_{i, t}+\beta_{4}^{\prime} \mathrm{SE}_{i, t}+\beta_{5}^{\prime} W \mathrm{SE}_{i, t}+\beta_{6}^{\prime} \mathrm{SP}_{i, t} \\
& +\beta_{7}^{\prime} W \mathrm{SP}_{i, t}+\beta_{8}^{\prime} E_{i, t-1}+\beta_{9}^{\prime} P_{i, t-1}+\sum_{e m p=1}^{K_{1}} \alpha_{e m p} X_{i, t-1}^{e m p}+v_{i, t}
\end{aligned}\right. \\
& \left\{\begin{aligned}
\mathrm{SP}_{i, t} & =\alpha_{0}^{\prime}+\alpha_{1}^{\prime} \mathrm{SE}_{i, t}+\alpha_{2}^{\prime} W \mathrm{SE}_{i, t}+\alpha_{3}^{\prime} W \mathrm{SP}_{i, t}+\alpha_{4}^{\prime} \mathrm{EP}_{i, t}+\alpha_{5}^{\prime} W \mathrm{EP}_{i, t}+\alpha_{6}^{\prime} \mathrm{EE}_{i, t} \\
& +\alpha_{7}^{\prime} W \mathrm{EE}_{i, t}+\alpha_{8}^{\prime} P_{i, t-1}+\alpha_{9}^{\prime} E_{i, t-1}+\sum_{p o p=1}^{K_{2}} \beta_{p o p} X_{i, t-1}^{p o p}+\mu_{i, t} \\
\mathrm{SE}_{i, t} & =\beta_{0}^{\prime}+\beta_{1}^{\prime} \mathrm{SP}_{i, t}+\beta_{2}^{\prime} W \mathrm{SP}_{i, t}+\beta_{3}^{\prime} W \mathrm{SE}_{i, t}+\beta_{4}^{\prime} \mathrm{EE}_{i, t}+\beta_{5}^{\prime} W \mathrm{EE}_{i, t}+\beta_{6}^{\prime} \mathrm{EP}_{i, t} \\
& +\beta_{7}^{\prime} W \mathrm{EP}_{i, t}+\beta_{8}^{\prime} E_{i, t-1}+\beta_{9}^{\prime} P_{i, t-1}+\sum_{p o p=1}^{K_{2}} \beta_{p o p} X_{i, t-1}^{p o p}+v_{i, t}
\end{aligned}\right.
\end{aligned}
$$

L'estimation du système d'équations simultanées permet, à un instant donné, de vérifier si c'est la population qui suit l'emploi ou si c'est l'emploi qui suit la population grâce aux paramètres $\alpha_{1}^{\prime}$ et $\beta_{1}^{\prime}$ des équations (7) et (8). Si $\alpha_{1}^{\prime}>0$ et $\beta_{1}^{\prime}=0$, on dit que la population suit l'emploi. À l'inverse, si $\alpha_{1}^{\prime}=0$ et $\beta_{1}^{\prime}>0$, c'est l'emploi qui suit la population. Enfin, si $\alpha_{1}^{\prime}>0$ et $\beta_{1}^{\prime}>0$, les évolutions de l'emploi et de la population s'influencent mutuellement.

\section{Méthode d'estimation}

Les variations de population et d'emploi au sein d'une zone donnée peuvent être mesurées de deux façons : comme l'évolution observée entre deux dates d'une part, comme la différence entre les mouvements d'entrées et les mouvements de sorties au cours de la période d'autre part. Pour faciliter l'interprétation de nos résultats et s'assurer de leur comparabilité, nous retenons la seconde définition pour ne mobiliser qu'un seul jeu de données. En estimant les trois modèles présentés ci-dessus (équations 5,7 et 8), nous pouvons étudier l'influence mutuelle des variations de la population et de l'emploi, la relation entre les entrées d'emploi et les entrées de population et, enfin, le lien entre les sorties d'emploi et les sorties de population. Cette nouvelle spécification permet non seulement de vérifier si les mouvements d'entrées et de sorties 
suivent la même dynamique, mais également de voir si ce sont plutôt les mouvements d'entrées ou les mouvements de sorties qui alimentent les variations de population et d'emploi. Nous distinguons ensuite les mouvements d'emploi selon qu'il s'agit de contrats temporaires (CDD) ou de contrats signés pour une durée indéterminée. Les mouvements de la population sont également distingués selon le degré de qualification des personnes.

Au total, nous estimons 18 modèles pour interroger la complexité de la relation entre les dynamiques de l'emploi et celles de la population : les trois systèmes d'équations (5), (7) et (8) sont d'abord estimés en considérant les mouvements d'emploi dans leur ensemble, puis en distinguant les CDD et les CDI (les résultats estimés sont présentés dans les tableaux 3A, 3B, 3C). Dans les tableaux 4A, 4B, 4C, les trois systèmes sont estimés en distinguant les mouvements d'emploi et ceux de population qualifiée (que nous rapprochons des professions et catégories socioprofessionnelles [PCS] des professions intermédiaires et cadres) ou peu qualifiée (qui correspond ici aux employés et ouvriers) selon qu'ils concernent des mouvements en CDD ou en CDI.

Nous réalisons ces différentes estimations grâce à la méthode GS3SLS (Generalized Spatial Three Stage Least Squares) développée par Harry H. KelejIAN et Ingmar R. PRUCHA (2004), qui permet de tenir compte de trois éléments :

- La simultanéité entre les dynamiques de la population et de l'emploi : les emplois sont plutôt créés dans des territoires où la population est abondante et qualifiée, mais les individus peuvent aussi décider d'emménager dans des zones où le marché local de l'emploi est dynamique.

- La dépendance spatiale entre les variables d'intérêt : les zones qui sont les plus attractives pour la population peuvent être proches géographiquement (et par exemple, leur proximité avec une aménité naturelle telle que la mer peut justifier leur attrait pour la population), tout comme il peut exister une concentration des zones de forte dynamique économique (suivant ainsi la logique d'agglomération).

- La dépendance spatiale entre les deux équations : un individu peut résider dans une zone et travailler dans une zone voisine, tout comme les débouchés économiques d'une entreprise ne se limitent pas au territoire où elle est implantée.

Nous menons notre étude à l'échelle des zones d'emploi de la France métropolitaine. Il s'agit d'un découpage géographique réalisé par l'Insee à partir de l'observation des déplacements quotidiens des individus, de telle sorte que, au sein de chaque zone, la majorité des individus y travaillent et y résident, et les entreprises sont susceptibles d'y trouver la main-d'œuvre nécessaire à leur activité. Partant de cette définition, nous supposons que les mobilités résidentielles qui ont lieu entre zones d'emploi sont des mobilités de « longue distance » et s'accompagnent le plus souvent d'un changement d'emploi pour les personnes concernées. Nous tenons compte dans ce modèle des effets de proximité géographique grâce à la matrice de voisinage. Nous avons retenu la matrice de contiguïté à l'ordre 1, qui semble la plus adaptée à l'objet de notre 
étude $^{3}$. Si les mobilités résidentielles entre zones d'emploi s'accompagnent la plupart du temps d'une mobilité professionnelle, il n'en demeure pas moins que certains individus peuvent déménager entre deux zones voisines sans pour autant changer d'emploi. En effet, nous supposons que certains individus peuvent travailler dans une zone d'emploi et résider dans une zone limitrophe. Afin de tenir compte de ces effets de débordement, nous considérons que chaque élément de la matrice $W$ vaut 1 si deux zones sont contiguës, et vaut 0 sinon. La matrice est ensuite standardisée par ligne, c'est-à-dire que la somme des éléments qui composent chaque ligne vaut 1 , ce qui permet de ne pas surestimer le poids du voisinage pour les unités qui sont entourées de nombreuses autres unités.

\section{Données mobilisées}

Pour mesurer les mouvements de population entre les zones d'emploi, nous mobilisons les données du Recensement de la population de 2013 sur les mobilités résidentielles. Les individus sont interrogés sur leur situation au $1^{\mathrm{er}}$ janvier 2013, en particulier sur leur lieu de résidence, leurs caractéristiques sociodémographiques, leur logement, etc., et sur leur lieu de résidence au $1^{\text {er }}$ janvier 2012. Nous retenons l'ensemble des individus âgés de 15 à 64 ans qui sont chefs de leur ménage ou conjoints, qu'ils soient en emploi salarié (secteur privé) ou au chômage ${ }^{4}$. Parce que nous les considérons comme les personnes décisionnaires de leurs trajectoires résidentielles (au total 8156306 individus), nous supposons que leur mobilité est le plus souvent choisie. Parmi eux, 15,9\% ont changé de logement entre 2012 et 2013 : 5,9\% ont changé de logement dans la même commune, $4,9 \%$ ont changé de commune au sein de la même zone d'emploi, et 5,1\% ont changé de zone d'emploi au sein de la France métropolitaine continentale ${ }^{5}$. Nous nous focalisons sur cette dernière partie de la population, qui représente au total 415958 individus.

Pour chaque zone d'emploi, nous calculons le nombre d'individus qui ont emménagé depuis une autre zone d'emploi (les flux d'entrée : $\mathrm{EP}_{\mathrm{i}, t}$ ) et le nombre d'individus qui ont déménagé vers une autre zone d'emploi (les flux de sortie : $\mathrm{SP}_{\mathrm{i}, t}$ ). Nous en déduisons la variation de la population, comme le résultat de la différence entre le nombre d'entrants et de sortants. Afin de tenir compte de l'hétérogénéité des

\footnotetext{
3. Cette intuition est confirmée par les tests de dépendance spatiale qui ont été réalisés préalablement sur chaque variable d'intérêt. Ceux-ci révèlent que, quels que soient les mouvements d'emploi et de population considérés, la matrice de contiguïté à l'ordre 1 est la plus à même de transcrire la dépendance géographique entre les unités spatiales par rapport à la matrice de distance inverse.

4. Nous excluons ainsi de notre étude les agriculteurs, travailleurs indépendants ou chefs d'entreprise, ainsi que les étudiants et les retraités, dont les comportements migratoires ont des logiques propres qui sont peu ou pas en lien avec les mouvements de main-d'œuvre.

5. On ne s'intéresse ici qu'aux déplacements qui ont lieu entre les zones d'emploi de France métropolitaine continentale ; on ne comptabilise pas les individus qui résidaient en Corse, dans les Outre-mer ou à l'étranger au $1^{\mathrm{er}}$ janvier 2012.
} 
comportements individuels selon la PCS, nous regroupons d'un côté les individus qui occupent les emplois les plus qualifiés (cadres, professions intermédiaires et intellectuelles) et d'un autre, les ouvriers et employés.

Nous étudions les flux de main-d'œuvre au cours de l'année 2012 à partir des fichiers des DMMO-EMMO. Les Déclarations mensuelles des mouvements de maind'œuvre constituent une obligation de déclaration des embauches et des résiliations de contrats pour les entreprises de plus de 50 salariés du secteur privé, hors agences de travail temporaire. Les entreprises de moins de 50 salariés sont quant à elles interrogées lors des Enquêtes sur les mouvements de main-d'œuvre. Nous calculons pour chaque zone d'emploi l'ensemble des mouvements d'entrées et de sorties enregistrés au cours de l'année 2012 et déterminons les entrées en emploi $\left(\mathrm{EE}_{\mathrm{i}, t}\right)$ et les sorties d'emploi $\left(\mathrm{SE}_{\mathrm{i}, t}\right)$ au cours de la période. Les DMMO-EMMO nous permettent également de distinguer les mouvements d'entrées et les mouvements de sorties selon le type de contrat (CDD ou CDI) et selon la profession et catégorie socioprofessionnelle du poste occupé (regroupés selon les mêmes modalités que les mouvements de population).

Enfin, nous mobilisons un grand nombre de sources de données pour contrôler des caractéristiques propres de chaque zone d'emploi, qui peuvent influencer le dynamisme de l'emploi d'une part, et l'évolution de la population d'autre part. Ces variables exogènes décrivent les caractéristiques de la population active, de l'emploi et du marché du logement à partir du Recensement de la population de 2013. Le revenu fiscal médian, mis à disposition par l'Insee dans le Fichier localisé social et fiscal (Filosofi), est extrait des déclarations d'imposition de 2012. L'occupation des sols est renseignée dans la base Corine Land Cover (CLC) de 2012 (la surface d'espace vert comme la proximité au littoral d'un territoire donné y sont détaillées). La base Eider, base de données régionales et départementales sur l'environnement, l'énergie, le transport, le logement et la construction, indique les conditions climatiques des départements de France et, notamment, le nombre de jours d'ensoleillement. Les données de la Direction générale des finances publiques (DGFIP) donnent des informations sur la taxation locale en 2012 (pour plus de détails sur la construction des variables de contrôle, voir tableau 1 en annexe).

\section{Résultats}

\section{Statistiques descriptives}

Parmi les individus de 15-64 ans retenus dans notre étude, on observe des écarts dans les comportements migratoires en fonction de leurs caractéristiques individuelles (tableau 1). Les taux de mobilité résidentielle des ouvriers, des employés et des professions intermédiaires sont relativement similaires (14\% environ). Le taux de mobilité des cadres est légèrement plus faible. Alors que les ouvriers et les employés déménagent plus souvent au sein de la même commune, les cadres et les professions 
TABLEAU 1 - La mobilité résidentielle par profession et catégorie socioprofessionnelle (PCS)

\begin{tabular}{lccccc}
\hline & $\begin{array}{c}\text { Cadres et } \\
\text { professions } \\
\text { intellectuelles } \\
\text { supérieures }\end{array}$ & $\begin{array}{c}\text { Professions } \\
\text { intermédiaires }\end{array}$ & Employés & Ouvriers & Ensemble \\
\hline Taux de mobilité & $12,9 \%$ & $14 \%$ & $14,5 \%$ & $14,4 \%$ & $14 \%$ \\
Mobilités « courtes » (en milliers) & 363 & 632 & 776 & 590 & 2361 \\
Mobilités « longues » (en milliers) & $64 \%$ & $69 \%$ & $73 \%$ & $77 \%$ & $71 \%$ \\
& 201 & 286 & 283 & 180 & 951 \\
\hline
\end{tabular}

* Les mobilités courtes correspondent aux déménagements au sein de la même zone d'emploi, et les mobilités longues aux déménagements entre zones d'emploi différentes.

Lecture : au cours de l'année 2012, 12,9\% des cadres ont changé de logement. Parmi eux, 64 \% ont déménagé au sein de la même commune ou dans des communes appartenant à la même zone d'emploi, et $36 \%$ ont changé de zone d'emploi.

Champ : personnes âgées de 15 à 64 ans qui sont chef de ménage ou conjoint, et qui occupent un emploi salarié ou sont à la recherche d'un emploi.

Source : Recensement de la population 2013.

intermédiaires sont plus enclins à réaliser des mobilités de longue distance, entre des zones d'emploi différentes. Ces résultats concordent avec ceux présentés dans le dernier rapport de l'Observatoire des territoires sur les mobilités résidentielles en France (BACCAÏNI et al., 2018), qui soulignent notamment que plus on est diplômé, plus on réalise des mobilités de longue distance, tandis que la mobilité de courte distance est moins segmentée.

Sans surprise, les entrées et sorties de CDD (respectivement $81 \%$ et $78 \%$ du total, tableau 2) sont plus nombreuses que les entrées et sorties de CDI (19\% et $22 \%$ ). Cependant, il existe des écarts importants selon les profils recrutés. Les mouvements de main-d'œuvre des cadres concernent le plus souvent des CDI (52\% des flux d'entrées et $54 \%$ des flux de sorties) alors que ceux des employés se traduisent massivement par des CDD, qui représentent $88 \%$ des entrées et $86 \%$ des sorties. Si les volumes de flux d'entrées et de sorties par type de contrat sont relativement similaires, ce n'est

TABLEAU 2 - Les mouvements de main-d'œuvre par type de contrat et par PCS

\begin{tabular}{cccccc}
\hline & $\begin{array}{c}\text { Cadres et } \\
\text { professions } \\
\text { intellectuelles } \\
\text { supérieures }\end{array}$ & $\begin{array}{c}\text { Professions } \\
\text { intermédiaires }\end{array}$ & Employés & Ouvriers & Ensemble \\
\hline Flux d'entrées & 380821 & 671986 & 1935008 & 1105483 & 4093298 \\
Entrées en CDD & $48 \%$ & $79 \%$ & $88 \%$ & $83 \%$ & $81 \%$ \\
Entrées en CDI & $52 \%$ & $21 \%$ & $12 \%$ & $17 \%$ & $19 \%$ \\
\hline Flux de sorties & 379809 & 692068 & 1912187 & 1117051 & 4101115 \\
Sorties de CDD & $46 \%$ & $75 \%$ & $86 \%$ & $77 \%$ & $78 \%$ \\
Sorties de CDI & $54 \%$ & $25 \%$ & $14 \%$ & $23 \%$ & $22 \%$ \\
\hline
\end{tabular}

Lecture : on comptabilise 380821 embauches (flux d'entrées) de cadres au cours de l'année 2012, dont $48 \%$ sont en CDD et $52 \%$ en CDI.

Champ : embauches et résiliations de contrats dans le secteur privé, hors contrats d'intérim.

Source : DMMO-EMMO 2012. 
pas le cas des mouvements d'emploi en CDI pour les ouvriers en 2012 : la part des sorties de CDI est nettement plus élevée que la part des entrées en CDI (respectivement $23 \%$ contre $17 \%$ ). Ces évolutions sont très liées à la dégradation de la conjoncture sur le marché du travail : la hausse du chômage en 2012 a particulièrement affecté les employés et ouvriers (pour plus de détails, GugGEMOS, VIDALENC, 2013).

\section{Résultats de l'analyse économétrique}

Les tableaux 3 (3A, 3B, 3C) et $4(4 \mathrm{~A}, 4 \mathrm{~B}, 4 \mathrm{C})$ synthétisent les résultats des estimations réalisées. Pour simplifier, nous ne présentons pas l'effet des variables de contrôle, et seuls les effets significatifs sont reportés. Les tableaux $3 \mathrm{~A}$ et $4 \mathrm{~A}$ correspondent à l'estimation des variations de population et d'emploi (modèle (5)), les tableaux 3B et 4B à l'estimation des entrées de population et d'emploi (modèle (7)), et les tableaux $3 \mathrm{C}$ et $4 \mathrm{C}$ à celle des sorties de population et d'emploi (modèle (8)). L'hétérogénéité des effets selon le type de mouvements d'emploi (CDD ou CDI) est présentée dans les tableaux 3, et la distinction des effets selon le type de PCS est introduite dans les tableaux 4 .

Les résultats de l'analyse spatiale révèlent peu d'effets de débordement entre les zones géographiques, la plupart des effets spatiaux n'étant pas significatifs ou de faible amplitude (tableaux 2A et 2B en annexe). Un fait saillant ressort : lorsqu'on les considère dans leur ensemble, les variations de population semblent suivre des logiques similaires de regroupement de zones (une hausse de la population des zones voisines entraîne une hausse de la population de la zone étudiée), alors que les variations d'emploi reflètent plutôt des effets d'agglomération, le dynamisme d'une zone donnée semblant se faire au détriment de ses voisines. Ces résultats sont cohérents avec les enseignements de la littérature : d'une part, on assiste à une opposition entre des zones attractives pour la population au Sud-Ouest de la France, alors que les zones les moins attractives se trouvent plutôt au Nord-Est du pays (ARNOULT, 2020 ; BACCAÏNI et al., 2018). D'autre part, la croissance de l'emploi est plus marquée dans les aires urbaines de plus de 200000 habitants puisque les fonctions liées au capital humain et aux gains de productivité tendent à s'y regrouper (CATIN, VAN HUFFEL, 2019), ce qui tend à concentrer les hausses d'emploi au sein de quelques zones.

Par ailleurs, l'analyse des variations d'emploi et de population dans leur ensemble (tableau 3A) montre que, globalement, l'emploi suit la population et la population suit l'emploi, puisque leurs évolutions s'influencent réciproquement. Le résultat est le même si on distingue les mouvements en CDD et les mouvements en CDI : les variations d'emploi et de population vont suivre une dynamique jointe (tableau $3 \mathrm{~A}$ ). La méta-analyse réalisée par Gerke J. HoogRSTA et ses co-auteurs (2017) a montré que la plupart des travaux basés sur ce modèle d'estimation de la variation de l'emploi et de la population arrivent aux mêmes conclusions lorsque l'analyse est menée à une échelle géographique agrégée, ce qui est ici notre cas. 
TABLEAU 3A - Estimation des variations de population et d'emploi pour l'ensemble des mouvements, les mouvements en CDD et les mouvements en CDI

\begin{tabular}{lcc|cc|cc}
\hline & \multicolumn{2}{c|}{ Ensemble } & \multicolumn{2}{c|}{ CDD } & \multicolumn{2}{c}{ CDI } \\
\hline & $\begin{array}{c}\text { Variations } \\
\text { de population }\end{array}$ & $\begin{array}{c}\text { Variations } \\
\text { d'emploi }\end{array}$ & $\begin{array}{c}\text { Variations } \\
\text { de population }\end{array}$ & $\begin{array}{c}\text { Variations } \\
\text { d'emploi }\end{array}$ & $\begin{array}{c}\text { Variations } \\
\text { de population }\end{array}$ & $\begin{array}{c}\text { Variations } \\
\text { d'emploi }\end{array}$ \\
\hline \multirow{2}{*}{ Variations de population } & - & $1,11^{* * *}$ & & $0,20^{* * *}$ & & $0,33^{* * *}$ \\
& & $(0,131)$ & - & $(0,018)$ & - & $(0,036)$ \\
Variations d'emploi & $0,14^{* * *}$ & & $1,24^{* * *}$ & & $0,74^{* * *}$ & - \\
\hline
\end{tabular}

Notes : significativité au seuil de $10 \%(*) ; 5 \%(* *)$ et $1 \%(* * *)$, les écarts-types sont notés entre parenthèses.

Les variables de contrôle (annexe, tableau 1) ont été intégrées dans l'analyse mais ne sont pas reportées ; seuls les résultats significatifs des variables d'intérêt sont présentés.

Lecture : lorsqu'on considère les flux d'emploi et de population dans leur ensemble, une hausse de la population entraîne une hausse de l'emploi, et une hausse de l'emploi entraîne une hausse de la population.

Champ : individus de 15 à 64 ans, chefs de ménage ou conjoints, hors chefs d'entreprise, indépendants, retraités et étudiants, et mouvements de main-d'œuvre en CDD et CDI.

Source : Recensement de la population 2013, DMMO-EMMO 2012, CLC 2012, DGFIP 2012 et Filosofi 2012.

Pour autant, une relation plus complexe se dessine lorsque l'on distingue les mouvements d'entrées des mouvements de sorties. Lorsque l'on s'intéresse aux mouvements dans leur ensemble, les entrées de population et les entrées d'emploi s'influencent mutuellement (tableau 3B), alors que seules les sorties de population entraînent des sorties d'emploi (les sorties d'emploi n'entraînent pas de sorties de population, voir tableau 3C). On peut supposer que les comportements migratoires mettent plus de temps à s'adapter à des changements dans l'emploi que l'inverse : un choc négatif sur l'emploi aura des répercussions sur les mobilités résidentielles qui

TABLEAU 3B - Estimation des entrées de population et d'emploi pour l'ensemble des mouvements, les mouvements en CDD et les mouvements en CDI

\begin{tabular}{|c|c|c|c|c|c|c|}
\hline & \multicolumn{2}{|c|}{ Ensemble } & \multicolumn{2}{|c|}{ CDD } & \multicolumn{2}{|c|}{ CDI } \\
\hline & $\begin{array}{c}\text { Entrées } \\
\text { de population }\end{array}$ & $\begin{array}{l}\text { Entrées } \\
\text { d'emploi }\end{array}$ & $\begin{array}{c}\text { Entrées } \\
\text { de population }\end{array}$ & $\begin{array}{l}\text { Entrées } \\
\text { d'emploi }\end{array}$ & $\begin{array}{c}\text { Entrées } \\
\text { de population }\end{array}$ & $\begin{array}{l}\text { Entrées } \\
\text { d'emploi }\end{array}$ \\
\hline Entrées de population & - & $\begin{array}{l}0,96^{* * *} \\
(0,134)\end{array}$ & - & $\begin{array}{l}0,15^{* * * *} \\
(0,021)\end{array}$ & - & $\begin{array}{l}0,26^{* * * *} \\
(0,035)\end{array}$ \\
\hline Entrées d'emploi & $\begin{array}{l}0,23^{* * *} \\
(0,039)\end{array}$ & - & n.s. & - & $\begin{array}{l}0,42^{* * *} \\
(0,100)\end{array}$ & - \\
\hline Sorties de population & $\begin{array}{l}0,93^{* * *} \\
(0,047)\end{array}$ & $\begin{array}{c}-1,35^{* * *} \\
(0,157)\end{array}$ & $\begin{array}{l}0,73^{* * *} \\
(0,054)\end{array}$ & $\begin{array}{c}-0,19^{* * * *} \\
(0,023)\end{array}$ & $\begin{array}{l}0,93^{* * *} \\
(0,046)\end{array}$ & $\begin{array}{c}-0,31^{* * * *} \\
(0,050)\end{array}$ \\
\hline Sorties d'emploi & $\begin{array}{c}-0,23^{* * * *} \\
(0,041)\end{array}$ & $\begin{array}{l}1,03^{* * *} \\
(0,010)\end{array}$ & n.s. & $\begin{array}{l}1,02^{* * *} \\
(0,005)\end{array}$ & $\begin{array}{c}-0,53^{* * * *} \\
(0,101)\end{array}$ & $\begin{array}{l}0,77^{* * * *} \\
(0,040)\end{array}$ \\
\hline
\end{tabular}

Notes : significativité au seuil de $10 \%(*) ; 5 \%(* *)$ et $1 \%(* * *)$, et n.s. non significatif. Les écarts-types sont notés entre parenthèses. Les variables de contrôles (annexe, tableau 1) ont été intégrées dans l'analyse mais ne sont pas reportées ; seuls les résultats significatifs des variables d'intérêt sont présentés.

Lecture : lorsqu'on considère l'ensemble des mouvements sans distinguer le type de contrat de travail, une hausse des sorties d'emploi entraîne une baisse des entrées de population.

Champ : individus de 15 à 64 ans, chefs de ménage ou conjoints, hors chefs d'entreprise, indépendants, retraités et étudiants, et mouvements de main-d'œuvre en CDD et CDI.

Source : Recensement de la population 2013, DMMO-EMMO 2012, CLC 2012, DGFIP 2012 et Filosofi 2012. 
TABLEAU 3C - Estimation des sorties de population et d'emploi pour l'ensemble des mouvements, les mouvements en CDD et les mouvements en CDI

\begin{tabular}{|c|c|c|c|c|c|c|}
\hline & \multicolumn{2}{|c|}{ Ensemble } & \multicolumn{2}{|c|}{ CDD } & \multicolumn{2}{|c|}{ CDI } \\
\hline & $\begin{array}{c}\text { Sorties } \\
\text { de population }\end{array}$ & $\begin{array}{c}\text { Sorties } \\
\text { d'emploi }\end{array}$ & $\begin{array}{c}\text { Sorties } \\
\text { de population }\end{array}$ & $\begin{array}{c}\text { Sorties } \\
\text { d'emploi }\end{array}$ & $\begin{array}{c}\text { Sorties } \\
\text { de population }\end{array}$ & $\begin{array}{l}\text { Sorties } \\
\text { d'emploi }\end{array}$ \\
\hline Sorties de population & - & $\begin{array}{l}0,61^{* * *} \\
(0,220)\end{array}$ & - & $\begin{array}{l}0,25^{* * * *} \\
(0,030)\end{array}$ & - & $\begin{array}{l}0,62^{* * * *} \\
(0,056)\end{array}$ \\
\hline Sorties d'emploi & n.s. & - & n.s. & - & $\begin{array}{l}0,84^{* * *} \\
(0,113)\end{array}$ & - \\
\hline Entrées de population & $\begin{array}{l}0,69^{* * *} \\
(0,037)\end{array}$ & $\begin{array}{l}-0,30^{* *} \\
(0,134)\end{array}$ & $\begin{array}{l}0,69^{* * *} \\
(0,054)\end{array}$ & $\begin{array}{c}-0,11^{* * * *} \\
(0,019)\end{array}$ & $\begin{array}{l}0,61^{* * *} \\
(0,032)\end{array}$ & $\begin{array}{c}-0,32^{* * * *} \\
(0,032)\end{array}$ \\
\hline Entrées d'emploi & n.s. & $\begin{array}{l}0,93^{* * *} \\
(0,009)\end{array}$ & n.s. & $\begin{array}{l}0,97^{* * *} \\
(0,004)\end{array}$ & $\begin{array}{c}-0,58^{* * *} \\
(0,093)\end{array}$ & $\begin{array}{l}0,73^{* * *} \\
(0,038)\end{array}$ \\
\hline
\end{tabular}

Notes : significativité au seuil de $10 \%(*) ; 5 \%(* *)$ et $1 \%(* * *)$, et n.s. non significatif. Les écarts-types sont notés entre parenthèses. Les variables de contrôle (annexe, tableau 1) ont été intégrées dans l'analyse mais ne sont pas reportées ; seuls les résultats significatifs des variables d'intérêt sont présentés.

Lecture : lorsqu'on considère l'ensemble des mouvements sans distinguer le type de contrat de travail, les sorties de population entraînent des sorties d'emploi, mais les sorties d'emploi n'ont pas d'effet sur les sorties de population.

Champ : individus de 15 à 64 ans, chefs de ménage ou conjoints, hors chefs d'entreprise, indépendants, retraités et étudiants, et mouvements de main-d'œuvre en CDD et CDI.

Source : Recensement de la population 2013, DMMO-EMMO 2012, CLC 2012, DGFIP 2012 et Filosofi 2012.

ne seront pas immédiates. Ce résultat est peut-être en partie lié à la structure de nos données et à la fenêtre temporelle d'un an.

La distinction entre les CDD et les CDI dans l'analyse des flux d'entrées et de sorties permet en outre de mettre en évidence des résultats qui diffèrent selon le type de contrat de travail. Dans le modèle des « entrées » (tableau 3B) comme dans celui des « sorties » (tableau 3C), on observe une influence mutuelle entre population et emploi quand on se focalise sur les CDI. En revanche, seules les sorties de population entraînent des mouvements d'emploi en CDD (les sorties d'emploi n'entraînent pas de mouvements de population). Cela peut s'expliquer par le développement des activités de la sphère résidentielle avec l'évolution de la population ; en 2012, le recours aux CDD est particulièrement élevé dans le secteur de l'action sociale, ainsi que parmi les activités récréatives et sportives (PARAIRE, 2014). En d'autres termes, les emplois en CDD sont très liés aux mouvements de population, alors que les mouvements de population sont seulement influencés par les mouvements d'emploi en CDI.

Les tableaux 4A, 4B et 4C permettent d'affiner encore l'analyse des mouvements de population et d'emploi en distinguant, pour chaque type de contrat de travail, la PCS (professions intermédiaires et cadres d'une part, ouvriers et employés d'autre part). Ce regroupement repose en partie sur les statistiques descriptives présentées dans la partie précédente, et confirmées par les analyses économétriques, montrant que les individus exerçant des emplois de cadres (et professions intellectuelles) ou de professions intermédiaires changent plus souvent de zone d'emploi que les employés et les ouvriers. 
TABLEAU 4A - Estimation des variations de population et d'emploi selon les PCS et en distinguant les mouvements en CDD et en CDI

\begin{tabular}{|c|c|c|c|c|c|c|c|c|}
\hline & \multicolumn{4}{|c|}{ Mouvements d'emploi en CDD } & \multicolumn{4}{|c|}{ Mouvements d'emploi en CDI } \\
\hline & \multicolumn{2}{|c|}{$\begin{array}{l}\text { Cadres et professions } \\
\text { intermédiaires }\end{array}$} & \multicolumn{2}{|c|}{ Employés et ouvriers } & \multicolumn{2}{|c|}{$\begin{array}{l}\text { Cadres et professions } \\
\text { intermédiaires }\end{array}$} & \multicolumn{2}{|c|}{ Employés et ouvriers } \\
\hline & $\begin{array}{c}\text { Variations } \\
\text { de } \\
\text { population }\end{array}$ & $\begin{array}{l}\text { Variations } \\
\text { d'emploi }\end{array}$ & $\begin{array}{c}\text { Variations } \\
\text { de } \\
\text { population }\end{array}$ & $\begin{array}{l}\text { Variations } \\
\text { d'emploi }\end{array}$ & $\begin{array}{c}\text { Variations } \\
\text { de } \\
\text { population }\end{array}$ & $\begin{array}{l}\text { Variations } \\
\text { d'emploi }\end{array}$ & $\begin{array}{l}\text { Variations } \\
\text { de } \\
\text { population }\end{array}$ & $\begin{array}{l}\text { Variations } \\
\text { d'emploi }\end{array}$ \\
\hline $\begin{array}{l}\text { Variations } \\
\text { de population }\end{array}$ & - & n.s. & - & $\begin{array}{l}0,33^{* * * *} \\
(0,035)\end{array}$ & - & $\begin{array}{l}0,56^{* * * *} \\
(0,061)\end{array}$ & - & $\begin{array}{l}0,25^{* * * *} \\
(0,064)\end{array}$ \\
\hline $\begin{array}{l}\text { Variations } \\
\text { d'emploi }\end{array}$ & n.s. & - & n.s. & - & $\begin{array}{l}0,62^{* * *} \\
(0,111)\end{array}$ & - & n.s. & - \\
\hline
\end{tabular}

Notes : significativité au seuil de $10 \%(*) ; 5 \%(* *)$ et $1 \%(* * *)$, et n.s. non significatif. Les écarts-types sont notés entre parenthèses. Les variables de contrôle (annexe, tableau 1) ont été intégrées dans l'analyse mais ne sont pas reportées ; seuls les résultats significatifs des variables d'intérêt sont présentés.

Lecture : parmi les employés et les ouvriers, une hausse de la population entraîne une hausse des emplois en CDD, la hausse des emplois en CDD ne fait pas varier la population.

Champ : individus de 15 à 64 ans, chefs de ménage ou conjoints, hors chefs d'entreprise, indépendants, retraités et étudiants, et mouvements de main-d'œuvre en CDD et CDI.

Source : Recensement de la population 2013, DMMO-EMMO 2012, CLC 2012, DGFIP 2012 et Filosofi 2012.

TABLEAU 4B - Estimation des entrées de population et d'emploi selon les PCS et en distinguant les mouvements en CDD et en CDI

\begin{tabular}{|c|c|c|c|c|c|c|c|c|}
\hline & \multicolumn{4}{|c|}{ Mouvements d'emploi en CDD } & \multicolumn{4}{|c|}{ Mouvements d'emploi en CDI } \\
\hline & \multicolumn{2}{|c|}{$\begin{array}{l}\text { Cadres et professions } \\
\text { intermédiaires }\end{array}$} & \multicolumn{2}{|c|}{ Employés et ouvriers } & \multicolumn{2}{|c|}{$\begin{array}{c}\text { Cadres et professions } \\
\text { intermédiaires }\end{array}$} & \multicolumn{2}{|c|}{ Employés et ouvriers } \\
\hline & $\begin{array}{l}\text { Entrées de } \\
\text { population }\end{array}$ & $\begin{array}{l}\text { Entrées } \\
\text { d'emploi }\end{array}$ & $\begin{array}{l}\text { Entrées de } \\
\text { population }\end{array}$ & $\begin{array}{l}\text { Entrées } \\
\text { d'emploi }\end{array}$ & $\begin{array}{l}\text { Entrées de } \\
\text { population }\end{array}$ & $\begin{array}{l}\text { Entrées } \\
\text { d'emploi }\end{array}$ & $\begin{array}{l}\text { Entrées de } \\
\text { population }\end{array}$ & $\begin{array}{l}\text { Entrées } \\
\text { d'emploi }\end{array}$ \\
\hline $\begin{array}{l}\text { Entrées } \\
\text { de population }\end{array}$ & - & $\begin{array}{l}0,05^{* * *} \\
(0,017)\end{array}$ & - & $\begin{array}{l}0,32^{* * * *} \\
(0,037)\end{array}$ & - & $\begin{array}{l}0,33^{* * *} \\
(0,073)\end{array}$ & - & $\begin{array}{l}0,34^{* * *} \\
(0,056)\end{array}$ \\
\hline $\begin{array}{l}\text { Entrées } \\
\text { d'emploi }\end{array}$ & $\begin{array}{l}1,17^{* * *} \\
(0,339)\end{array}$ & - & n.s. & - & $\begin{array}{l}0,44^{* * * *} \\
(0,107)\end{array}$ & - & $\begin{array}{l}0,22^{* * * *} \\
(0,076)\end{array}$ & - \\
\hline $\begin{array}{l}\text { Sorties } \\
\text { de population }\end{array}$ & $\begin{array}{l}0,69^{* * * *} \\
(0,036)\end{array}$ & n.s. & $\begin{array}{l}0,81^{* * * *} \\
(0,035)\end{array}$ & $\begin{array}{c}-0,37^{* * * *} \\
(0,037)\end{array}$ & $\begin{array}{l}0,84^{* * * *} \\
(0,040)\end{array}$ & $\begin{array}{l}-0,46 \text { **** } \\
(0,056)\end{array}$ & $\begin{array}{l}0,77^{* * * *} \\
(0,034)\end{array}$ & $\begin{array}{l}-0,27^{* * *} \\
(0,059)\end{array}$ \\
\hline $\begin{array}{l}\text { Sorties } \\
\text { d'emploi }\end{array}$ & $\begin{array}{c}-1,19^{* * * *} \\
(0,342)\end{array}$ & $\begin{array}{l}1,02^{* * *} \\
(0,003)\end{array}$ & n.s. & $\begin{array}{l}1,00^{* * *} \\
(0,003)\end{array}$ & $\begin{array}{c}-0,42^{* * *} \\
(0,099)\end{array}$ & $\begin{array}{l}0,91^{* * *} \\
(0,026)\end{array}$ & $\begin{array}{l}-0,12^{*} \\
(0,061)\end{array}$ & $\begin{array}{l}0,64^{* * *} \\
(0,035)\end{array}$ \\
\hline
\end{tabular}

Notes : significativité au seuil de $10 \%(*) ; 5 \%(* *)$ et $1 \%(* * *)$, et n.s. non significatif. Les écarts-types sont notés entre parenthèses. Les variables de contrôles (annexe, tableau 1) ont été intégrées dans l'analyse mais ne sont pas reportées ; seuls les résultats significatifs des variables d'intérêt sont présentés.

Lecture : parmi les cadres et les professions intermédiaires, une hausse des entrées de population entraîne une hausse des entrées en CDD, et une hausse des entrées en CDD entraîne une hausse de la population.

Champ : individus de 15 à 64 ans, chefs de ménage ou conjoints, hors chefs d'entreprise, indépendants, retraités et étudiants, et mouvements de main-d'œuvre en CDD et CDI.

Source : Recensement de la population 2013, DMMO-EMMO 2012, CLC 2012, DGFIP 2012 et Filosofi 2012. 
TABLEAU 4C - Estimation des sorties de population et d'emploi selon les PCS et en distinguant les mouvements en CDD et en CDI

\begin{tabular}{|c|c|c|c|c|c|c|c|c|}
\hline & \multicolumn{4}{|c|}{ Mouvements d'emploi en CDD } & \multicolumn{4}{|c|}{ Mouvements d'emploi en CDI } \\
\hline & \multicolumn{2}{|c|}{$\begin{array}{l}\text { Cadres et professions } \\
\text { intermédiaires }\end{array}$} & \multicolumn{2}{|c|}{ Employés et ouvriers } & \multicolumn{2}{|c|}{$\begin{array}{l}\text { Cadres et professions } \\
\text { intermédiaires }\end{array}$} & \multicolumn{2}{|c|}{ Employés et ouvriers } \\
\hline & $\begin{array}{l}\text { Sorties de } \\
\text { population }\end{array}$ & $\begin{array}{l}\text { Sorties } \\
\text { d'emploi }\end{array}$ & $\begin{array}{l}\text { Sorties de } \\
\text { population }\end{array}$ & $\begin{array}{l}\text { Sorties } \\
\text { d'emploi }\end{array}$ & $\begin{array}{l}\text { Sorties de } \\
\text { population }\end{array}$ & $\begin{array}{l}\text { Sorties } \\
\text { d'emploi }\end{array}$ & $\begin{array}{l}\text { Sorties de } \\
\text { population }\end{array}$ & $\begin{array}{l}\text { Sorties } \\
\text { d'emploi }\end{array}$ \\
\hline $\begin{array}{l}\text { Sorties } \\
\text { de population }\end{array}$ & - & $\begin{array}{l}-0,04^{* *} \\
(0,018)\end{array}$ & - & $\begin{array}{l}0,39^{* * *} \\
(0,046)\end{array}$ & - & $\begin{array}{l}0,62^{* * * *} \\
(0,066)\end{array}$ & - & $\begin{array}{l}0,41^{* * *} \\
(0,082)\end{array}$ \\
\hline $\begin{array}{l}\text { Sorties } \\
\text { d'emploi }\end{array}$ & $\begin{array}{l}-0,92^{* *} \\
(0,365)\end{array}$ & - & n.s. & - & n.s. & - & n.s. & - \\
\hline $\begin{array}{l}\text { Entrées } \\
\text { de population }\end{array}$ & $\begin{array}{l}0,80^{* * *} \\
(0,042)\end{array}$ & n.s. & $\begin{array}{l}0,88^{* * *} \\
(0,038)\end{array}$ & $\begin{array}{c}-0,25^{* * *} \\
(0,035)\end{array}$ & $\begin{array}{l}0,82^{* * *} \\
(0,042)\end{array}$ & $\begin{array}{c}-0,39^{* * *} \\
(0,059)\end{array}$ & $\begin{array}{l}0,85^{* * * *} \\
(0,042)\end{array}$ & $\begin{array}{l}-0,16^{* * *} \\
(0,061)\end{array}$ \\
\hline $\begin{array}{l}\text { Entrées } \\
\text { d'emploi }\end{array}$ & $\begin{array}{c}0,88^{* *} \\
(0,362)\end{array}$ & $\begin{array}{l}0,97^{* * *} \\
(0,003)\end{array}$ & n.s. & $\begin{array}{l}0,99^{* * *} \\
(0,003)\end{array}$ & $\begin{array}{l}-0,27^{* *} \\
(0,121)\end{array}$ & $\begin{array}{l}0,95^{* * *} \\
(0,027)\end{array}$ & n.s. & $\begin{array}{l}0,81^{* * *} \\
(0,046)\end{array}$ \\
\hline
\end{tabular}

Notes : significativité au seuil de $10 \%(*) ; 5 \%(* *)$ et $1 \%(* * *)$, et n.s. non significatif. Les écarts-types sont notés entre parenthèses. Les variables de contrôles (annexe, tableau 1) ont été intégrées dans l'analyse mais ne sont pas reportées ; seuls les résultats significatifs des variables d'intérêt sont présentés.

Lecture : parmi les cadres et les professions intermédiaires, une hausse des sorties de population entraîne une baisse des sorties d'emploi en CDD, et une hausse des entrées en CDD entraîne une hausse des sorties de population.

Champ : individus de 15 à 64 ans, chefs de ménage ou conjoints, hors chefs d'entreprise, indépendants, retraités et étudiants, et mouvements de main-d'œuvre en CDD et CDI.

Source : Recensement de la population 2013, DMMO-EMMO 2012, CLC 2012, DGFIP 2012 et Filosofi 2012.

Les résultats consignés reflètent l'hétérogénéité de la relation entre la mobilité résidentielle et la croissance locale de l'emploi en fonction de la PCS. Pour les ouvriers et les employés, c'est l'emploi qui suit la population plutôt que l'inverse : les évolutions de l'emploi en CDD comme en CDI sont très sensibles aux évolutions de la population, qu'elles soient mesurées en variations (tableau 4A), à partir des mouvements d'entrées (tableau 4B) ou de sorties (tableau 4C). Une hausse de la population entraîne une hausse de l'emploi, mais l'inverse ne se vérifie que pour les entrées en CDI. Puisque les employés et les ouvriers réalisent moins souvent des mobilités résidentielles de longue distance, les entreprises se rapprochent de la main-d'œuvre nécessaire à leur activité et les emplois sont créés dans les bassins dynamiques du point de vue de la population.

Les résultats sont moins homogènes lorsque l'on s'intéresse aux cadres et aux professions intermédiaires : les variations de population et d'emploi en CDD ne sont pas liées, alors que les variations de population et d'emploi en CDI s'influencent mutuellement (tableau 4A). Lorsque l'on décompose l'analyse des variations entre mouvements d'entrées et de sorties, l'absence de relation entre les évolutions de la population et de l'emploi en CDD s'explique par une influence réciproque positive dans les mouvements d'entrées (tableau 4B) face à une influence réciproque négative dans les mouvements de sorties (tableau 4C) : les entrées d'emploi en CDD entraînent des entrées de population, et réciproquement, alors que les sorties d'emploi en CDD entraînent une baisse des sorties de population et inversement. Cette moindre relation entre les dynamiques de la population et les dynamiques de l'emploi en CDD pour les 
cadres et les professions intermédiaires peut s'expliquer par leurs choix de localisation résidentielle. Si les entrées d'emploi en CDD augmentent, les cadres et les professions intermédiaires sont prêts à emménager dans la zone pour se rapprocher de leur emploi. À l'inverse, si les sorties d'emploi en CDD augmentent, cela ne se traduit pas par des sorties de population, puisqu'ils vont écouler les possibilités d'emploi des zones géographiques voisines, quitte à accroître leurs déplacements domicile-travail avant de déménager. Le peu de recul temporel sur les données pourrait en partie expliquer ce résultat.

L'analyse des mouvements de CDI confirme la propension à la mobilité plus forte des cadres et des professions intellectuelles : l'emploi suit la population et la population suit l'emploi, lorsqu'ils sont mesurés en variation ou à partir des flux d'entrées. En d'autres termes, une hausse de l'emploi (tableau 4A) ou des entrées d'emploi (tableau 4B) en CDI entraîne respectivement une hausse de la population ou des entrées de population de cadres et de professions intermédiaires, alors que ce n'était pas le cas pour les employés et ouvriers : une hausse de l'emploi en CDI n'entraîne pas de hausse de la population (tableau 4A). De nouveau, le résultat n'est pas significatif pour les mouvements de sorties (tableau 4c) : pour les ouvriers et les employés, une sortie des emplois en CDI n'a pas d'effet sur les sorties de population.

Dans cet article, nous estimons un modèle classique de l'économétrie spatiale (le modèle de G. A. CARLino et E. S. MiLls [1987]) en étudiant non pas les variations ou les taux de croissance de la population et de l'emploi comme c'est le cas habituellement, mais en mobilisant directement les flux de population et d'emploi, c'est-à-dire les mouvements d'entrées et de sorties des zones d'emploi. Les données que nous utilisons nous permettent de distinguer les mouvements d'emploi (secteur privé) et de population selon les types de contrat de travail et les niveaux de qualification des emplois. Nos résultats viennent compléter les connaissances sur les sources de la croissance locale de l'emploi et de la population. Les plus saillants d'entre eux montrent que les entrées de population et les entrées d'emploi s'influencent mutuellement alors que seules les sorties de population entraînent des sorties d'emploi. Pour expliquer cela, on peut supposer que les comportements migratoires mettent plus de temps à s'adapter à des changements dans l'emploi que l'inverse : un choc négatif sur l'emploi aura des répercussions sur les mobilités résidentielles qui ne seront pas immédiates.

De plus, dans le modèle des « entrées » comme dans le modèle des « sorties », on note une influence réciproque entre population et emploi quand on se focalise sur les CDI dans leur ensemble. En revanche, seuls les mouvements de population entraînent des mouvements d'emploi en CDD. En d'autres termes, les emplois en CDD sont très liés aux mouvements de population, alors que les mouvements de population sont seulement influencés par les mouvements d'emploi en CDI. 
Enfin, nos analyses donnent à penser que les ouvriers et les employés ont tendance à effectuer des mobilités résidentielles de courte distance : leur propension à la mobilité est plus faible que celle des cadres et des professions intermédiaires (en cas de choc négatif sur l'emploi). Du fait d'un ancrage territorial plus fort que celui des autres catégories, ils semblent d'abord chercher à écouler l'ensemble des possibilités d'emploi à proximité de leur commune d'origine, avant de chercher un emploi en dehors de leur zone d'emploi.

Ces résultats confortent le fait que la compréhension des comportements migratoires ne peut se cantonner à l'étude de la population et des emplois dans leur ensemble. D'une part, les trajectoires résidentielles des ménages diffèrent selon leurs profils socioéconomiques. D'autre part, l'idée selon laquelle les individus déménageraient pour l'emploi paraît un peu forte et peu réaliste dans le cas de contrats courts. En effet, nos résultats confirment que ce sont les mouvements d'emploi en CDI qui impulsent les migrations résidentielles, mais pas ceux en CDD.

L'attractivité résidentielle et l'attractivité économique sont fortement liées, mais l'emploi suit la population plus que l'inverse. L'attractivité d'un territoire repose donc sur sa capacité à attirer la population et à offrir à ses habitants un cadre de vie qui leur convienne. Mais si l'emploi en CDI peut influencer les trajectoires résidentielles des personnes, les politiques de recrutements des entreprises et le contexte institutionnel en matière de protection de l'emploi ont un effet non négligeable sur les choix de localisation résidentielle et les disparités observées entre les territoires.

En prolongement de ce travail, il serait intéressant d'étudier s'il existe des effets multiplicatifs entre la dynamique des emplois en CDI et celle des emplois en CDD. Si les travaux menés par S. ØSTBYE et ses co-auteurs (2018) montrent que la croissance locale des emplois qualifiés et celle des emplois non qualifiés s'entraînent l'une l'autre, on peut faire l'hypothèse d'un même lien entre emplois permanents et temporaires. En effet, au sein des zones où l'activité économique est la plus prospère, la croissance des emplois en CDI pourrait entraîner une augmentation des contrats temporaires et inversement. L'investigation de cette hypothèse pourra faire l'objet de futurs travaux qui permettraient de tester si les dynamiques de l'emploi ont un impact sur les inégalités spatiales, selon que les contrats signés permettent, ou non, à la population de se projeter de façon durable au sein des territoires.

\section{BibliogRAPHIE}

Abildtrup J., Hilal M., Piguet V., Schmitt B. (2018), “Determinants of Local Population Growth and Economic Development in France between 1990 and 2006", Revue d'économie régionale et urbaine, vol. 2018, $\mathrm{n}^{\circ}$ 1, pp. 91-128.

Abildtrup J., Piguet V., Schmitt B. (2012), "Does the Agro-Food Industry Impact on Changes in Regional Employment and Population? The Case of Denmark", Food Economics, vol. 9, $\mathrm{n}^{\mathrm{o}} 3$, pp. 145-156. 
ARnoult É. (2020), « Migration résidentielle et croissance locale de l'emploi : une analyse des zones d'emploi de France métropolitaine », Revиe économique, vol. 71, n 1, pp. 83-107. Arnoult É., Duhautois R. (2019), “Workers and People Flows in France: Is There a Link?", Economics Bulletin, vol. 39, n 1, pp. 540-546.

Authier J.-Y (dir.), Bidet J., Collet A., Gilbert P., Steinmetz H. (2010), État des lieux sur les trajectoires résidentielles, La Défense, Plan urbanisme, construction architecture (PUCA).

BACCAÏNI B. (dir.), GARNIER M., HARAN L. (2018), Les Mobilités résidentielles en France. Tendances et impacts territoriaux. Rapport 2018 de l'Observatoire des territoires de 2018, Paris, Commissariat général à l'égalité des territoires.

BoARnet M. G. (1994), “An Empirical Model of Intrametropolitan Population and Employment Growth”, Papers in Regional Science, vol. 73, n 2, pp. 135-152.

BRIARD K. (2019), «Quels liens entre mobilité résidentielle et situation professionnelle ? », Dares analyses, $\mathrm{n}^{\circ} 15$.

Brueckner J. K., Thisse J.-F., Zenou Y. (1999), "Why is Central Paris Rich and Downtown Detroit Poor? An Amenity-Based Theory", European Economic Review, vol. 43, n 1 , pp. 91-107.

CARlino G. A., Mills E. S. (1987), “The Determinants of County Growth”, Journal of Regional Science, vol. 27, $\mathrm{n}^{\circ} 1$, pp. 39-54.

Catin M., Van Huffel C. (2019), «Disparités de croissance d'emploi des aires urbaines françaises et processus de métropolisation. Le rôle des fonctions métropolitaines, productives et des revenus extérieurs », Revue d'économie régionale et urbaine, vol. 2019, nº 4, pp. 749-779.

DEBRAND T., TAFFIN C. (2005), « Les facteurs structurels et conjoncturels de la mobilité résidentielle depuis 20 ans », Économie et Statistique, n ${ }^{\circ}$ 381-382, pp. 125-146.

de GraAfF T., van Oort F. G., Florax R. (2012), "Regional Population-Employment Dynamics across Different Sectors of the Economy", Journal of Regional Science, vol. 52, n 1, pp. 60-84.

Gebremariam G. H., Gebremedhin T. G., SchaefFer P. V., Jackson R. W. (2008), "Modeling Regional Growth Spillovers: An Analysis of Employment Growth, Migration Behavior, Local Public Services and Household Income in Appalachia", Virginia Polytechnic Institute and State University Working Paper, ${ }^{\circ}$ e07-13.

GobiLlon L. (2001), « Emploi, logement et mobilité résidentielle », Économie et Statistique, $\mathrm{n}^{\mathrm{o}} 349-350$, pp. 77-98.

Guggemos F., VidAlenC J. (2013), « Une photographie du marché du travail en 2012 », Insee Première, $\mathrm{n}^{\circ} 1466$.

HelPMAn E., KRUGMAN P. R. (1985), Market Structure and Foreign Trade: Increasing Returns, Imperfect Competition and the International Economy, Brighton, Wheatsheaf.

Hoogstra G. J., van Dijk J., Florax R. (2005), "Do Jobs Follow People or People Follow jobs? A Meta-Analysis of Carlino-Mills Studies", 45th Congress of the European Regional Science Association, Amsterdam, The Netherlands. 
Hoogstra G. J., van Dijk J., Florax R. J. (2017), “Do Jobs Follow People or People Follow Jobs? A Meta-Analysis of Carlino-Mills Studies", Spatial Economic Analysis, vol. 12, n 4, pp. 357-378.

Huu Phe H., Wakely P. (2000), "Status, Quality and the Other Trade-Off: Towards a New Theory of Urban Residential Location”, Urban Studies, vol. 37, n 1, pp. 7-35.

JEAN P. (2008), «Économie résidentielle et productive : le choix des territoires », Le Quatre pages Insee Aquitaine, $\mathrm{n}^{\mathrm{o}} 175$.

Kelejian H. H., Prucha I. R. (2004), "Estimation of Simultaneous Systems of Spatially Interrelated Cross Sectional Equations", Journal of Econometrics, vol. 118, n 1-2, pp. 27-50.

Kim J. H., Pagliara F., Preston J. (2005), “The Intention to Move and Residential Location Choice Behaviour”, Urban Studies, vol. 42, n 9, pp. 1621-1636.

Krugman P., Venables A. J. (1990), "Integration and the Competitiveness of Peripheral Industry", in Bliss C., Braga de Macedo J. (eds.), Unity with Diversity in the European Community: The Community's Southern Frontier, Cambridge, Cambridge University Press, pp. 56-77.

MiLin K. (2018), « CDD, CDI : comment évoluent les embauches et les ruptures depuis 25 ans ?», Dares analyses, $\mathrm{n}^{\circ} 026$.

Østbye S., Moilanen M., Tervo H., Westerlund O. (2018), “The Creative Class: Do Jobs Follow People or Do People Follow Jobs?”, Regional Studies, vol. 52, nº 6, pp. 745-755.

PARAIRE X. (2014), « Les mouvements de main-d'œuvre en 2012. Recul des recrutements en CDI dans tous les secteurs », Dares analyses, $\mathrm{n}^{\circ} 003$.

Puga D. (1999), “The Rise and Fall of Regional Inequalities", European Economic Review, vol. 43, n ${ }^{\circ}$, pp. 303-334. 


\section{ANNEXE}

\section{TABLEAU 1 - Description des variables de contrôle utilisées}

\begin{tabular}{|c|c|c|}
\hline Variable & Source* & Description \\
\hline Taux d'encadrement & $R P 2013$ & $\begin{array}{l}\text { (cadres + professions intermédiaires)/(ouvriers + employés) } \\
=\text { information sur le nombre de qualifiés rapporté au nombre de peu } \\
\text { qualifiés }\end{array}$ \\
\hline Chômeurs & $R P 2013$ & nombre de chômeurs \\
\hline Revenu médian & Filosofi 2012 & revenu médian des ménages (échelle commune : moyenne pondérée) \\
\hline Maisons & $R P 2013$ & nombre de maisons parmi le parc de logements \\
\hline Logements récents & $R P 2013$ & nombre de logements construits depuis moins de dix ans \\
\hline Densité de population & $R P 2013$ & nombre d'habitants/surface de la zone d'emploi \\
\hline Taux de taxe d'habitation & DGFIP 2013 & $\begin{array}{l}\text { moyenne pondérée des taux de taxe d'habitation des communes de la } \\
\text { zone d'emploi considérée }\end{array}$ \\
\hline Espaces verts & CLC 2013 & surface occupée par les espaces verts de la zone d'emploi considérée \\
\hline Frontière & auteurs & $\begin{array}{l}\text { dichotomique = } 1 \text { si la zone d'emploi est située à la frontière du pays, } \\
=0 \text { sinon }\end{array}$ \\
\hline Littoral & auteurs & dichotomique $=1$ si la zone d'emploi est située sur le littoral, $=0$ sinon \\
\hline Emplois dans l'industrie & $R P 2013$ & nombre d'emplois du secteur industriel \\
\hline $\begin{array}{l}\text { Emplois dans le commerce } \\
\text { et les services }\end{array}$ & $R P 2013$ & nombre d'emplois dans le commerce ou les services \\
\hline
\end{tabular}

* RP 2013 : Recensement de la population de 2013 (Insee) ;

Filosofi 2012 : Fichier localisé social et fiscal (Insee) ;

DGFIP 2012 : données de la Direction générale des finances publiques de 2012 ;

CLC 2012 : base Corine Land Cover de 2012. 
TABLEAU 2A - Effets spatiaux sur l'estimation des variations, des entrées et des sorties d'emploi et de population pour l'ensemble des mouvements, les mouvements en CDD et les mouvements en CDI

\begin{tabular}{|c|c|c|c|c|c|c|}
\hline & \multicolumn{2}{|c|}{ Ensemble } & \multicolumn{2}{|c|}{ CDD } & \multicolumn{2}{|c|}{ CDI } \\
\hline & $\begin{array}{l}\text { Variation } \\
\text { de la } \\
\text { population }\end{array}$ & $\begin{array}{l}\text { Variation } \\
\text { de } \\
\text { l'emploi }\end{array}$ & $\begin{array}{c}\text { Variation } \\
\text { de la } \\
\text { population }\end{array}$ & $\begin{array}{l}\text { Variation } \\
\text { de } \\
\text { l'emploi }\end{array}$ & $\begin{array}{c}\text { Variation } \\
\text { de la } \\
\text { population }\end{array}$ & $\begin{array}{l}\text { Variation } \\
\text { de } \\
\text { l'emploi }\end{array}$ \\
\hline $\begin{array}{l}\text { W*variation } \\
\text { de la population }\end{array}$ & $\begin{array}{c}0,09^{\text {*** }} \\
(0,039)\end{array}$ & n.s. & n.s. & n.s. & n.s. & n.s. \\
\hline \multirow[t]{2}{*}{$\begin{array}{l}\text { W*variation } \\
\text { de l'emploi }\end{array}$} & n.s. & $\begin{array}{l}-0,20^{*} \\
(0,116)\end{array}$ & $\begin{array}{l}-0,30^{* * * *} \\
(0,078)\end{array}$ & $\begin{array}{c}0,76^{* *} \\
(0,035)\end{array}$ & $\begin{array}{c}0,18^{* * *} \\
(0,079)\end{array}$ & n.s. \\
\hline & $\begin{array}{l}\text { Entrées de } \\
\text { population }\end{array}$ & $\begin{array}{l}\text { Entrées } \\
\text { d'emploi }\end{array}$ & $\begin{array}{l}\text { Entrées de } \\
\text { population }\end{array}$ & $\begin{array}{l}\text { Entrées } \\
\text { d'emploi }\end{array}$ & $\begin{array}{l}\text { Entrées de } \\
\text { population }\end{array}$ & $\begin{array}{l}\text { Entrées } \\
\text { d'emploi }\end{array}$ \\
\hline $\begin{array}{l}\text { W*entrées de population } \\
W^{*} \text { entrées d'emploi } \\
W^{*} \text { sorties de population } \\
W^{*} \text { sorties d'emploi }\end{array}$ & $\begin{array}{l}\text { n.s. } \\
\text { n.s. } \\
\text { n.s. } \\
\text { n.s. }\end{array}$ & $\begin{array}{l}\text { n.s. } \\
\text { n.s. } \\
\text { n.s. } \\
\text { n.s. }\end{array}$ & $\begin{array}{l}\text { n.s. } \\
\text { n.s. } \\
\text { n.s. } \\
\text { n.s. }\end{array}$ & $\begin{array}{l}\text { n.s. } \\
\text { n.s. } \\
\text { n.s. } \\
\text { n.s. }\end{array}$ & $\begin{array}{l}\text { n.s. } \\
\text { n.s. } \\
\text { n.s. } \\
\text { n.s. }\end{array}$ & $\begin{array}{l}\text { n.s. } \\
\text { n.s. } \\
\text { n.s. } \\
\text { n.s. }\end{array}$ \\
\hline W*sorties d'emploi & $\begin{array}{l}\text { Sorties de } \\
\text { population }\end{array}$ & $\begin{array}{l}\text { Sorties } \\
\text { d'emploi }\end{array}$ & $\begin{array}{l}\text { Sorties de } \\
\text { population }\end{array}$ & $\begin{array}{l}\text { Sorties } \\
\text { d'emploi }\end{array}$ & $\begin{array}{l}\text { Sorties de } \\
\text { population }\end{array}$ & $\begin{array}{l}\text { Sorties } \\
\text { d'emploi }\end{array}$ \\
\hline $\mathrm{W}^{*}$ sorties de population & n.s. & $\begin{array}{l}-0,47^{*} \\
(0,239)\end{array}$ & n.s. & $\begin{array}{l}-0,06^{*} \\
(0,030)\end{array}$ & n.s. & n.s. \\
\hline W*sorties d'emploi & n.s. & n.s. & n.s. & n.s. & n.s. & n.s. \\
\hline $\mathrm{W}^{*}$ entrées de population & n.s. & n.s. & $\begin{array}{l}-0,08^{*} \\
(0,044)\end{array}$ & $\begin{array}{l}0,04^{* * * *} \\
(0,019)\end{array}$ & n.s. & n.s. \\
\hline W*entrées d'emploi & n.s. & n.s. & n.s. & n.s. & n.s. & n.s. \\
\hline
\end{tabular}

Notes : significativité au seuil de $10 \%(*) ; 5 \%(* *)$ et $1 \%(* * *)$, et $n . s$. non significatif. Les écarts-types sont notés entre parenthèses. Les variables de contrôle (annexe, tableau 1) ont été intégrées dans l'analyse mais ne sont pas reportées ; seuls les résultats significatifs des variables d'intérêt sont présentés.

Lecture : lorsqu'on considère les mouvements de population et d'emploi dans leur ensemble, une hausse de la variation de la population dans les zones géographiques voisines entraîne une baisse de la population dans la zone d'emploi considérée. À l'inverse, une hausse de l'emploi dans les zones géographiques voisines entraîne une baisse de l'emploi dans la zone géographique donnée.

Champ : individus de 15 à 64 ans, chefs de ménage ou conjoints, hors chefs d'entreprise, indépendants, retraités et étudiants, et mouvements de main-d'œuvre en CDD et CDI.

Source : Recensement de la population 2013, DMMO-EMMO 2012, CLC 2012, DGFIP 2012 et Filosofi 2012. 
TABLEAU 2B - Effets spatiaux sur l'estimation des variations, des entrées et des sorties d'emploi et de population selon les PCS pour l'ensemble des mouvements, les mouvements en CDD et les mouvements en CDI

\begin{tabular}{|c|c|c|c|c|c|c|c|c|}
\hline & \multicolumn{4}{|c|}{ Mouvements d'emploi en CDD } & \multicolumn{4}{|c|}{ Mouvements d'emploi en CDI } \\
\hline & \multicolumn{2}{|c|}{$\begin{array}{c}\text { Cadres et professions } \\
\text { intermédiaires }\end{array}$} & \multicolumn{2}{|c|}{ Employés et ouvriers } & \multicolumn{2}{|c|}{$\begin{array}{l}\text { Cadres et professions } \\
\text { intermédiaires }\end{array}$} & \multicolumn{2}{|c|}{ Employés et ouvriers } \\
\hline & $\begin{array}{l}\text { Variation } \\
\text { de la } \\
\text { population }\end{array}$ & $\begin{array}{l}\text { Variation } \\
\text { de } \\
\text { l'emploi }\end{array}$ & $\begin{array}{l}\text { Variation } \\
\text { de la } \\
\text { population }\end{array}$ & $\begin{array}{l}\text { Variation } \\
\text { de } \\
\text { l'emploi }\end{array}$ & $\begin{array}{l}\text { Variation } \\
\text { de la } \\
\text { population }\end{array}$ & $\begin{array}{l}\text { Variation } \\
\text { de } \\
\text { l'emploi }\end{array}$ & $\begin{array}{l}\text { Variation } \\
\text { de la } \\
\text { population }\end{array}$ & $\begin{array}{l}\text { Variation } \\
\text { de } \\
\text { l'emploi }\end{array}$ \\
\hline $\begin{array}{l}\text { W*variation } \\
\text { de la population }\end{array}$ & $\begin{array}{c}0,14^{* *} \\
(0,061)\end{array}$ & $\begin{array}{l}0,05^{* * *} \\
(0,018)\end{array}$ & n.s. & n.s. & n.s. & n.s. & n.s. & n.s. \\
\hline \multirow[t]{2}{*}{$\begin{array}{l}\text { W*variation } \\
\text { de l'emploi }\end{array}$} & n.s. & n.s. & n.s. & n.s. & n.s. & $\begin{array}{l}0,27^{* *} \\
(0,110)\end{array}$ & n.s. & n.s. \\
\hline & $\begin{array}{l}\text { Entrées de } \\
\text { population }\end{array}$ & $\begin{array}{l}\text { Entrées } \\
\text { d'emploi }\end{array}$ & $\begin{array}{l}\text { Entrées de } \\
\text { population }\end{array}$ & $\begin{array}{l}\text { Entrées } \\
\text { d'emploi }\end{array}$ & $\begin{array}{l}\text { Entrées de } \\
\text { population }\end{array}$ & $\begin{array}{l}\text { Entrées } \\
\text { d'emploi }\end{array}$ & $\begin{array}{l}\text { Entrées de } \\
\text { population }\end{array}$ & $\begin{array}{l}\text { Entrées } \\
\text { d'emploi }\end{array}$ \\
\hline $\begin{array}{l}\text { W*entrées } \\
\text { de population }\end{array}$ & n.s. & n.s. & n.s. & n.s. & $\begin{array}{c}0,21^{*} \\
(0,125)\end{array}$ & n.s. & n.s. & n.s. \\
\hline $\begin{array}{l}\text { W*entrées } \\
\text { d'emploi }\end{array}$ & n.s. & n.s. & n.s. & n.s. & n.s. & n.s. & n.s. & n.s. \\
\hline $\begin{array}{l}\text { W*sorties } \\
\text { de population }\end{array}$ & n.s. & n.s. & n.s. & n.s. & n.s. & n.s. & n.s. & n.s. \\
\hline \multirow[t]{2}{*}{$\begin{array}{l}\text { W*sorties } \\
\text { d'emploi }\end{array}$} & n.s. & n.s. & $\begin{array}{l}-0,23^{* *} \\
(0,102)\end{array}$ & n.s. & $\begin{array}{l}-0,21^{*} \\
(0,126)\end{array}$ & n.s. & n.s. & n.s. \\
\hline & $\begin{array}{l}\text { Sorties de } \\
\text { population }\end{array}$ & $\begin{array}{l}\text { Sorties } \\
\text { d'emploi }\end{array}$ & $\begin{array}{l}\text { Sorties de } \\
\text { population }\end{array}$ & $\begin{array}{l}\text { Sorties } \\
\text { d'emploi }\end{array}$ & $\begin{array}{l}\text { Sorties de } \\
\text { population }\end{array}$ & $\begin{array}{l}\text { Sorties } \\
\text { d'emploi }\end{array}$ & $\begin{array}{l}\text { Sorties de } \\
\text { population }\end{array}$ & $\begin{array}{l}\text { Sorties } \\
\text { d'emploi }\end{array}$ \\
\hline $\begin{array}{l}\text { W*sorties } \\
\text { de population }\end{array}$ & n.s. & $\begin{array}{c}0,03^{*} \\
(0,018)\end{array}$ & n.s. & $\begin{array}{l}-0,10^{* *} \\
(0,044)\end{array}$ & n.s. & n.s. & n.s. & n.s. \\
\hline $\begin{array}{l}\text { W*sorties } \\
\text { d'emploi }\end{array}$ & n.s. & n.s. & n.s. & n.s. & n.s. & n.s. & n.s. & n.s. \\
\hline $\begin{array}{l}\mathrm{W}^{*} \text { entrées } \\
\text { de population }\end{array}$ & n.s. & $\begin{array}{l}-0,03^{*} \\
(0,016)\end{array}$ & n.s. & n.s. & n.s. & n.s. & n.s. & n.s. \\
\hline $\begin{array}{l}\text { W*entrées } \\
\text { d'emploi }\end{array}$ & n.s. & n.s. & n.s. & n.s. & n.s. & n.s. & n.s. & n.s. \\
\hline
\end{tabular}

Notes : significativité au seuil de $10 \%(*) ; 5 \%(* *)$ et $1 \%(* * *)$, et n.s. non significatif. Les écarts-types sont notés entre parenthèses. Les variables de contrôles (annexe, tableau 1) ont été intégrées dans l'analyse mais ne sont pas reportées ; seuls les résultats significatifs des variables d'intérêt sont présentés.

Lecture : lorsqu'on considère les mouvements de population et d'emploi de cadres et de professions intermédiaires, une hausse de la population dans les zones voisines entraîne une hausse de la population et une hausse de l'emploi en CDD dans la zone considérée. Champ : individus de 15 à 64 ans, chefs de ménage ou conjoints, hors chefs d'entreprise, indépendants, retraités et étudiants, et mouvements de main-d'œuvre en CDD et CDI.

Source : Recensement de la population 2013, DMMO-EMMO 2012, CLC 2012, DGFIP 2012 et Filosofi 2012. 